\title{
Personalisierte Therapie im Vorteil
}

Individuelle Tumormerkmale erhalten immer größere therapeutische Bedeutung. Für die Behandlung des fortgeschrittenen NSCLC wurde ein oraler Inhibitor der Anaplastischen-Lymphom-Kinase (ALK) neu zugelassen.

Genotypische Untersuchungen konnten bei verschiedenen Tumoren aktivierende Mutationen, sogenannte Treibermutationen (driver mutations) identifizieren, die das Tumorwachstum bestimmen. Beim nicht-kleinzelligen Lungenkarzinom (NSCLC) sind dies z.B. aktivierende Mutationen des EGFR (Epidermal Growth Factor Receptor), KRAS-Mutationen oder die ALK-Translokation, erklärte Jürgen Wolf, Köln. Diese Treibermutationen bieten Chancen für individuell adaptierte Therapien, die auch das Gesamtüberleben substanziell verbessern können, betonte Wolf. So verlängerte Erlotinib das Überleben bei unselektionierten Patienten mit NSCLC um zwei Monate, bei einer EGFRMutation aber um zwei bis drei Jahre. Ähnlich effektiv ist Crizotinib (Xalkoriø) bei vorbehandelten Patienten mit fortgeschrittenem, ALK-positivem Tumor. Crizotinib wurde am 23. Oktober von der Europäischen Kommission in dieser Indikation zugelassen.

Grundlage der personalisierten Medizin ist eine hochwertige molekulare Dia- gnostik, bei der oft mit nur wenigen Tumorzellen eine große Anzahl von Genen gleichzeitig sequenziert werden soll, erklärte Reinhard Büttner, Köln. Diese Präzisionsdiagnostik könnten nur spezialisierte Zentren leisten. Deshalb sind neue Modelle wie das „Netzwerk Genomische Medizin Lungenkrebs"von Kliniken und niedergelassenen Onkologen im Großraum Köln/Bonn erforderlich: Die Genotypisierung wird am Institut für Pathologie der Universität Köln durchgeführt. Danach erhalten die Patienten heimatnah eine personalisierte Therapie oder sie können - wenn diese Therapie nicht unmittelbar zur Verfügung steht - in eine Studie eingeschlossen werden. Günter Springer

Pressekonferenz „Unverzichtbar: Molekulare Diagnostik als Basis für die personalisierte Therapie beim Lungenkrebs", Köln, 30.08.2012; Veranstalter: Pfizer Pharma

\section{Wechsel der antiangiogenen Substanz nach First-Line-Versagen?}

Eine neue Substanz könnte antiangiogen vorbehandelten Patienten, die dennoch ein Rezidiv entwickelten, einen Überlebensvorteil bieten.

Für die Second-Line-Therapie des metastasierten kolorektalen Karzinoms (mCRC) befindet sich die antiangiogene Substanz Aflibercept im Zulassungsverfahren. Sie bietet Rezidivpatienten (auch Patienten mit Angiogenesehemmer-Vortherapie) die Chance auf einen signifikanten Überlebensvorteil. Derzeit liegt für Aflibercept eine positive Empfehlung der europäischen Zulassungsbehörde vor.

Aflibercept besteht aus den Bindungsstellen der beiden Rezeptoren des vaskulären endothelialen Wachstumsfaktors VEGF-R1 und VEGF-R2. So kann es nicht nur den VEGF-A-Liganden, sondern auch VEGF-B und den PIGF (Plazenta Growth Factor) abfangen. Alle drei Wachstumsfaktoren beeinflussen die Tumorangiogenese.

Dirk Arnold, Hamburg, schließt einen Vorteil durch den Wechsel der antiangiogenen Substanz gegenüber der Weiterbehandlung mit derselben Substanz nicht aus. In der zulassungsrelevanten randomisierten Phase-III-Studie [Tabernero J et al. EJC 2011;47(2): 6LBA] verlängerte die Kombi- nation aus FOLFIRI (5-FU/Folsäure, Irinotecan) plus Aflibercept das mediane Gesamtüberleben der bereits vorbehandelten Patienten mit mCRC signifikant, ohne das Nebenwirkungsprofil klinisch relevant zu erhöhen. Auch die mit dem Angiogenesehemmer Bevacizumab vorbehandelten $\mathrm{Pa}$ - tienten profitierten Second-line von Aflibercept, betonte Arnold. Birgit Pohlmann

Satellitensymposium „Choosing an optimal fit; exploring treatments for the patient with mCRC" im Rahmen der ESMO-Jahrestagung, Wien, 28.09.2012; Veranstalter: Sanofi-Aventis

NSCLC mit EGFR-Mutation

\section{Afatinib: auch mehr Lebensqualität}

Bei fortgeschrittenem nicht-kleinzelligem Lungenkarzinom (NSCLC) und positivem EGFR-Mutationsstatus verbessert Afatinib das progressionsfreie Überleben und wirkt auch effektiver gegen tumorbedingte Beschwerden.

Die Ergebnisse der LUX-Lung 3-Studie unterstreichen die hohe Wirksamkeit des Tyrosinkinasehemmers Afatinib, erläuterte Vera Hirsh, Montreal, Kanada. Sie zeigen, dass sich auch die tumorbedingten Beschwerden unter Afatinib signifikant besser zurückbilden und deutlich länger stabil bleiben als unter der Chemothera- pie. Die Patienten im Afatinib-Arm geben weniger Husten, Kurzatmigkeit und Schmerzen an [Sequist L et al. ESMO 2012, Poster P1229-PD]. Die Patienten haben einen besseren globalen Gesundheitszustand sowie Vorteile im physischen, emotionalen, kognitiven und sozialen Bereich und im Rollenverhalten. Dies ist laut Hirsh auch Ausdruck der besseren Symptomkontrolle durch Afatinib. Birgit Pohlmann

Pressekonferenz "Lung Cancer: what lies beneath the data" im Rahmen der ESMO-Jahrestagung, Wien, 28.09.2012; Veranstalter: Boehringer Ingelheim 